PREPARED FOR THE U.S. DEPARTMENT OF ENERGY, UNDER CONTRACT DE-AC02-76CH03073

PPPL-3749

PPPL-3749

UC-70

FIRE, A Next Step Option for Magnetic Fusion

by

D.M. Meade

September 2002

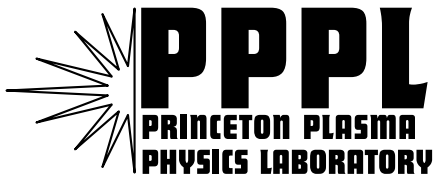

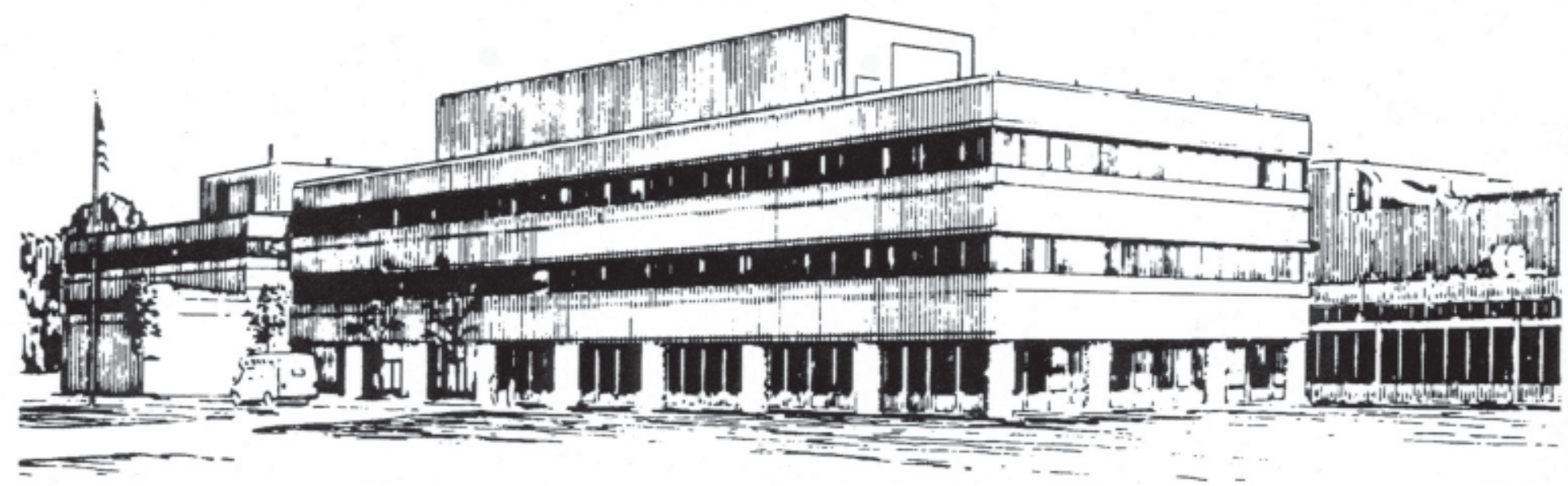

PRINCETON PLASMA PHYSICS LABORATORY PRINCETON UNIVERSITY, PRINCETON, NEW JERSEY 


\section{PPPL Reports Disclaimer}

This report was prepared as an account of work sponsored by an agency of the United States Government. Neither the United States Government nor any agency thereof, nor any of their employees, makes any warranty, express or implied, or assumes any legal liability or responsibility for the accuracy, completeness, or usefulness of any information, apparatus, product, or process disclosed, or represents that its use would not infringe privately owned rights. Reference herein to any specific commercial product, process, or service by trade name, trademark, manufacturer, or otherwise, does not necessarily constitute or imply its endorsement, recommendation, or favoring by the United States Government or any agency thereof. The views and opinions of authors expressed herein do not necessarily state or reflect those of the United States Government or any agency thereof.

\section{Availability}

This report is posted on the U.S. Department of Energy's Princeton Plasma Physics Laboratory Publications and Reports web site in Fiscal Year 2002. The home page for PPPL Reports and Publications is: http://www.pppl.gov/pub_report/

DOE and DOE Contractors can obtain copies of this report from:

U.S. Department of Energy

Office of Scientific and Technical Information

DOE Technical Information Services (DTIS)

P.O. Box 62

Oak Ridge, TN 37831

Telephone: (865) 576-8401

Fax: (865) 576-5728

Email: reports@adonis.osti.gov

This report is available to the general public from:

National Technical Information Service

U.S. Department of Commerce

5285 Port Royal Road

Springfield, VA 22161

Telephone: 1-800-553-6847 or

(703) 605-6000

Fax: (703) 321-8547

Internet: http://www.ntis.gov/ordering.htm 


\title{
FIRE, A NEXT STEP OPTION FOR MAGNETIC FUSION
}

\author{
D. M. MEADE \\ Princeton Plasma Physics Laboratory \\ P.O. Box 451 \\ Princeton, NJ, 08540 USA \\ 609-243-3301(T), 609-243-3315(F), dmeade@pppl.gov
}

\begin{abstract}
The next major frontier in magnetic fusion physics is to explore and understand the strong non-linear coupling among confinement, MHD stability, self-heating, edge physics and waveparticle interactions that is fundamental to fusion plasma behavior. The Fusion Ignition Research Experiment (FIRE) design study has been undertaken to define the lowest cost facility to attain, explore, understand and optimize magnetically confined fusion-dominated plasmas. FIRE is envisioned as an extension of the existing advanced tokamak program that could lead to an attractive magnetic fusion reactor. FIRE activities have focused on the physics and engineering assessment of a compact, high-field tokamak with the capability of achieving $\mathrm{Q} \approx 10$ in the Elmy $\mathrm{H}$-mode for a duration of $\sim 1.5$ plasma current redistribution times (skin times) during an initial burning plasma science phase, and the flexibility to add advanced tokamak hardware (e.g., lower hybrid current drive) later. The configuration chosen for FIRE is similar to that of ARIES-RS, the U. S. Fusion Power Plant study utilizing an advanced tokamak reactor. The key "advanced tokamak" features are: strong plasma shaping, double-null pumping divertors, low toroidal field ripple $(<0.3 \%)$, internal control coils and space for wall stabilization capabilities. The reference design point is $\mathrm{R}_{\mathrm{o}}=2.14 \mathrm{~m}$, a $=0.595 \mathrm{~m}, \mathrm{~B}_{\mathrm{t}}\left(\mathrm{R}_{\mathrm{o}}\right)=10 \mathrm{~T}, \mathrm{I}_{\mathrm{p}}=7.7 \mathrm{MA}$ with a flat top time of $20 \mathrm{~s}$ for $150 \mathrm{MW}$ of fusion power. The baseline magnetic fields and pulse lengths can be provided by wedged $\mathrm{BeCu} / \mathrm{OFHC}$ toroidal field (TF) coils and OFHC poloidal field (PF) coils that are pre-cooled to $80 \mathrm{~K}$ prior to the pulse and allowed to warm up to $373 \mathrm{~K}$ at the end of the pulse. A longer term goal of FIRE is to explore advanced tokamak regimes sustained by non-inductive current drive (e.g. lower hybrid current drive) at high fusion gain $(\mathrm{Q}>5)$ for a duration of 1 to 3 current redistribution times.
\end{abstract}

\section{Keywords:}

fusion, burning plasma experiment, ignition, tokamak, alpha particles 


\section{Introduction}

Magnetic fusion is technically ready to proceed to the next stage of fusion research, the study of burning plasmas dominated by the fusion process. A team consisting of scientists and engineers from more than 15 institutions in the U.S. fusion community is designing an advanced tokamak device, known as the Fusion Ignition Research Experiment (FIRE), whose primary mission is to attain, explore, understand and optimize magnetically confined fusiondominated plasmas $[1,2]$. The FIRE pre-conceptual design activities have been undertaken with the objective of finding the minimum size (cost) device to achieve the essential burning plasma science goals. This work is managed through the Virtual Laboratory for Technology and is funded by the U. S. Department of Energy. The FIRE experiment is envisioned as part of an international multi-machine strategy that consists of burning plasma experiment(s), very long-pulse non-burning experiments in advanced configurations (advanced tokamak, advanced stellarator, etc,) and fusion technology development facilities including a high fluence fusion materials irradiation facility. These individual facilities could be in the $\sim \$ 1 \mathrm{~B}$ range or less, and could be led by parties sited around the world.

\section{General Physics Requirements for a Burning Plasma Experiment, FIRE}

The first goal of FIRE is to carry out burning plasma experiments to address confinement, MHD stability, fast alpha physics and alpha heating and edge plasma issues expected in fusion reactor scale plasmas. For these experiments alpha heating must dominate the plasma dynamics. The goal for FIRE is to achieve plasmas where the alpha-heating is $\geq 2$ times the plasma heating power supplied externally. This corresponds to plasmas with an alpha heating fraction of $\mathrm{f}_{\alpha}=\mathrm{P}_{\text {alpha }} / \mathrm{P}_{\text {heating }} \geq 2 / 3$ or $\mathrm{Q}=\mathrm{P}_{\text {fusion }} / \mathrm{P}_{\text {ext-heating }} \geq 10$. Ignition would not be precluded under slightly more optimistic physics.

FIRE is also being designed to study burning plasmas in advanced configurations in a later phase as an extension of the existing advanced tokamak program. For these experiments, it will be desirable to study regimes that are bootstrap current dominated, $f_{b s}=I_{b s} / I_{p} \geq 50 \% \quad\left(\beta_{N}\right.$ $\geq 2.6)$ with the possibility of exploring $\mathrm{f}_{\mathrm{bs}}$ up to $75 \%\left(\beta_{\mathrm{N}} \sim 3.6\right)$. The higher $\beta$ regime would require strong plasma shaping and stabilization of the $n=1$ kink by a conducting first wall or feedback.

The pulse duration is a very important requirement for these experiments and should be specified in terms of the natural plasma time scales such as: $\tau_{\mathrm{E}}$ the energy confinement time, $\tau_{\mathrm{He}}$ the He ash confinement time and $\tau_{\mathrm{CR}}$ the plasma current redistribution time. The goal for FIRE pulse duration is: $>10 \tau_{\mathrm{E}}$ for pressure profile evolution, $>4 \tau_{\mathrm{He}}$ for alpha ash transport and burn control, and $\sim 2 \tau_{\mathrm{CR}}$ for plasma current profile evolution in advanced regimes.

\section{Optimization of a Burning Plasma Experiment}

A systems study was undertaken to find the minimum size burning plasma needed to satisfy the physics requirements discussed above. This study was specialized for inductively-driven tokamaks with $\mathrm{TF}$ and $\mathrm{PF}$ coils that are pre-cooled to $\mathrm{LN}_{2}$ temperature, and then heated adiabatically during the pulse. The systems code includes constraints for stress, resistive and 
nuclear heating of the coils and volt-sec requirements. The geometry can be chosen to have TF and PF coils unlinked with continuous conductors as in FIRE, or linked with a jointed TF conductors as in low aspect ratio tokamaks (ST). The code optimizes the allocation of the space in the inner coil stack between the free standing ohmic solenoid and the wedged TF coil. The confinement was taken to be H-mode with ITER98 $(y, 2)$ scaling [3]. For these studies, the plasma density profile had a small peaking of $\mathrm{n}(0) /\langle\mathrm{n}\rangle=1+\alpha_{\mathrm{n}}=1.2, \mathrm{n} / \mathrm{n}_{\mathrm{GW}} \leq$ 0.75 , and modest temperature profile peaking of $\mathrm{T}(0) /\langle\mathrm{T}\rangle=1+\alpha_{\mathrm{T}}=2.0$ where $\alpha_{\mathrm{n}}$ and $\alpha_{\mathrm{T}}$ are the exponents of parabolic squared profile functions for density and temperature. The plasma impurities consist of helium ash with the $\tau_{\mathrm{He}}=5 \tau_{\mathrm{E}}$ and $3 \% \mathrm{Be}$ impurities. The systems code varied the major radius, $\mathrm{R}$, and aspect ratio, $\mathrm{A}$, with $\mathrm{H}(\mathrm{y}, 2)=1.1, \kappa_{95}=1.8, \mathrm{q}_{\mathrm{cyl}}=3.1$ and $\mathrm{P}_{\text {fusion }}=150 \mathrm{MW}$ to obtain plasmas with $\mathrm{Q} \sim 10$ and $20 \mathrm{~s}$ burn time. For these constraints, the smallest size device to achieve the burning plasma requirements for a cryogenically-cooled inductively driven tokamak with unlinked $\mathrm{TF} / \mathrm{PF}$ coils has a shallow minimum around $\mathrm{A} \approx$ $3.6, \mathrm{~B} \approx 10 \mathrm{~T}$ and $\mathrm{R} \approx 2.1 \mathrm{~m}$ as shown in Fig. 1 . The normalized burn time measured in plasma current redistribution times, $\tau_{\mathrm{J}}=\tau_{\mathrm{burn}} / \tau_{\mathrm{CR}}$, increases significantly as the aspect ratio is increased as shown in Fig. 1. The minimum aspect ratio that satisfies the physics requirement of $2 \tau_{\mathrm{J}}$ is $\mathrm{A} \geq 3.4$. The advanced tokamak feature of significant bootstrap current is also enhanced at higher aspect ratios. Indeed, the fusion power plant design studies based on advanced tokamak scenarios, such as ARIES-RS [4] and ASSTR [5], have chosen A = 4 and relatively high magnetic fields of $8.2 \mathrm{~T}$ and $11 \mathrm{~T}$, respectively. These objectives have lead to the choice of $\mathrm{A}=3.6$ for FIRE which is higher than the aspect ratios chosen for other burning plasma experiments such as ITER-FEAT [3] and IGNITOR [6] which have A $=3.1$ and 2.8, respectively.

\section{FIRE Configuration and General Parameters}

The FIRE engineering activities have focused on the physics and engineering assessment of a compact, copper-alloy conductor, high-field tokamak with the capability of achieving $\mathrm{Q} \approx 10$ in the Elmy $\mathrm{H}$-mode for a duration of $\sim 1.5$ plasma current redistribution times during an initial burning plasma science phase. FIRE will also have the flexibility and remote handling capability to add advanced tokamak hardware (e.g., lower hybrid current drive launchers mounted in the midplane ports) in a later upgrade phase. FIRE has chosen a configuration with a highly shaped plasma, double-null divertor and aspect ratio $\approx 3.6$, similar to that of other advanced tokamak designs such as TPX, KSTAR and ARIES-RS. The key "advanced tokamak" features are: segmented central solenoid for flexibility and strong plasma shaping, double-null pumped divertors, low toroidal field ripple $(<0.3 \%)$, internal control coils and space for wall stabilization capabilities. The reference design point is $R_{0}=2.14 \mathrm{~m}, \mathrm{a}=0.595$ $\mathrm{m}, \mathrm{B}_{\mathrm{t}}\left(\mathrm{R}_{\mathrm{o}}\right)=10 \mathrm{~T}, \mathrm{I}_{\mathrm{p}}=7.7 \mathrm{MA}$ with a flat top time of $20 \mathrm{~s}$ for $150 \mathrm{MW}$ of fusion power with the parameters and cross-section shown in Table I and Fig. 2. The plasma heating for the FIRE reference operating mode would be provided by $20 \mathrm{MW}$ of ion cyclotron (ICRF) in the frequency range of $80-120 \mathrm{MHz}$, utilizing 4 ports [7]. Port space is reserved for an additional $10 \mathrm{MW}$ ICRF power upgrade if required. The primary heating modes will be $\mathrm{He} 3$ minority and second harmonic tritium near $10 \mathrm{~T}$, or H-minority and second harmonic deuterium near $6.5 \mathrm{~T}$. A total of $20 \mathrm{MW}$ of LHCD is reserved as an upgrade for advanced tokamak (AT) operation and NTM control. Plasma fueling would be provided by high-field side and vertical 
pellet injection for core fueling with gas and low-field side pellet injection for edge fueling.

\section{Plasma Performance Projections for Elmy H-Mode Operation}

The operating modes, physics issues and physics design guidelines for projecting burning plasma performance in FIRE are similar to those for ITER-FEAT [3]. The natural operating regime for FIRE is well matched to the existing H-mode database, and FIRE can access the density range from $0.3<\mathrm{n} / \mathrm{n}_{\mathrm{GW}}<1.0$ through a combination of pellet fueling and divertor pumping [1,2]. This flexibility is important for investigating the onset of alpha-driven modes at the lower densities, and to optimize the edge plasma for confinement studies and efficient divertor operation at higher densities. The performance of FIRE can be projected by selecting JET data in the International Tokamak Data Base, DB03v5, with parameters similar to FIRE, namely $\beta_{\mathrm{N}} \geq 1.7, \mathrm{Z}_{\mathrm{eff}}<2.0, \kappa>1.7$ and $2.7<\mathrm{q}_{95}<3.5$. The average $\mathrm{H}(\mathrm{y}, 2)$ and density profile peaking, $\mathrm{n}(0) /\langle\mathrm{n}\rangle_{\mathrm{V}}$ for these data (Fig 3) were found to be 1.1 and 1.2, respectively. This is consistent with the analysis of JET H-mode data presented by Cordey et al [8] that found that the average $\mathrm{H}$ factor increases with plasma cross-section triangularity, and has a maximum as the density is varied at $\mathrm{n} / \mathrm{n}_{\mathrm{GW}} \approx 0.6$ as shown in Fig. 3. A 0 -D power balance code was used to calculate the Q-value in FIRE as a function of $\mathrm{H}$-factor as shown in Fig. 4. The density profile was assumed to have $\mathrm{n}(0) /\langle\mathrm{n}\rangle_{\mathrm{V}}=1.2$ (x points) or 1.5 ( $\Delta$ points) with $3 \%$ Be and self-consistent alpha ash accumulation for $\tau_{\mathrm{He}}=5 \tau_{\mathrm{E}}$. The slightly more peaked profiles are consistent with density profiles observed during neutral beam heating in DIII-D H-modes and more recently on JET. FIRE would aim to achieve this modestly peaked profile using pellet fueling in ICRF heated discharges. More R\&D is needed on existing experiments, such as CMod to develop the scenario. The assumption of modest temperature profile peaking $\mathrm{T}(0) /\langle\mathrm{T}\rangle=2.0$, based on post sawtooth profiles in 1.5 D simulations, is more conservative than the ITER FEAT assumption of $\mathrm{T}(0) /\langle\mathrm{T}\rangle=3.15$. On this basis, FIRE would be expected to achieve fusion dominated plasmas with $f_{\alpha}=P_{\text {alpha }} / P_{\text {heating }} \geq 2 / 3(Q \geq 10)$ for elmy H-mode operation. Typical plasma parameters for a $\mathrm{Q}=10$ plasma in FIRE are given in Table I.

Physics based models using marginal stability transport such as GLF23 [9] have been used to predict burning plasma performance in FIRE. These models depend sensitively on the value of the H-mode temperature pedestal which is projected to be higher for plasmas with strong shaping (triangularity), and pedestal density low relative to the Greenwald density. Application of GLF23 to FIRE by Kinsey [9] has shown that it is possible to attain $Q \approx 10$ with edge temperature pedestals in the range of 3 to $4 \mathrm{keV}$. A next step experiment, such as FIRE, would provide a strong test of these models and improve their capability for predicting reactor plasma performance.

A simulation of the elmy H-mode regime using the 1 1/2 -D Tokamak Simulation Code (TSC) [10], with transport modeled by matching $\mathrm{H}(\mathrm{y}, 2)=1.1$ and $\mathrm{n}(0) /\langle\mathrm{n}\rangle_{\mathrm{V}}=1.2$, indicates that FIRE can access the H-Mode and sustain alpha-dominated plasmas for a duration $>20 \tau_{\mathrm{E}}$, $>4 \tau_{\mathrm{He}}$ and $\sim 1.5 \tau_{\text {skin }}$ as shown in Fig. 5. In addition, time is provided for plasma startup and controlled shutdown to avoid plasma disruptions. The burn phase is sufficiently long to allow the study of plasma profile evolution due to alpha heating, accumulation of alpha ash, testing of techniques for burn control and the initial studies of plasma current evolution due to alpha 
heating.

Neoclassical tearing modes (NTMs) pose a potential threat to the achievement of the required $\beta_{\mathrm{N}}$ values in tokamak burning-plasma experiments such as FIRE, since the polarizationcurrent stabilization model predicts that the critical $\beta_{\mathrm{N}}$ for their onset scales like $\rho_{\mathrm{i}}{ }^{*}$. The value of $\rho_{i}^{*}$ in FIRE is intermediate between that in present-day tokamaks such as JET and that in ITER-FEAT, and NTMs might arise in FIRE for the reference values of $\beta_{\mathrm{N}}(1.5-2.0)$. For this reason, NTM suppression by feedback-modulated LHCD is being evaluated. Encouraging results on the complete stabilization of NTMs using lower hybrid current drive have been obtained on COMPASS-D [11]. Calculations with a LHCD model in the TSC code have shown that a $12-15 \mathrm{MW} 4.6 \mathrm{GHz}$ system with 50/50 on/off modulation should be capable of suppressing the $\mathrm{m} / \mathrm{n}=3 / 2$ mode up to $\beta_{\mathrm{N}} \approx 2.0$ on FIRE. The possibility of using electron cyclotron current drive (ECCD) in the range of $170 \mathrm{GHz}$ for advanced tokamak modes at $\sim 6.5 \mathrm{~T}$ is being investigated.

\section{Advanced Modes in FIRE}

The standard regime in FIRE without wall stabilization is limited by kink instabilities to $\beta_{\mathrm{N}}$ $<3$ and bootstrap factions, $\mathrm{f}_{\mathrm{bs}} \leq 50 \%$. Exploitation of advanced tokamak regimes requires stabilization of the low $n$ kinks as recently demonstrated on DIII-D [12]. If the $n=1$ kink could be stabilized by a conducting wall or feedback in FIRE, then advanced tokamak regimes with $\beta_{\mathrm{N}} \leq 3.6$ and $\mathrm{f}_{\mathrm{bs}} \leq 75 \%$ would be possible. Studies using the VALEN [12] code have shown that feedback stabilization coils placed on the horizontal port plug faces would be very effective at stabilizing kink modes, and would allow access to $\beta_{\mathrm{N}}$ up to 3.8 [13]. TSC has been used to determine the current drive, plasma heating power and energy confinement required to dynamically access these advanced regimes in a burning plasma [14]. The example shown in Fig. 5 has $\mathrm{B}=8.5 \mathrm{~T}, \mathrm{I}_{\mathrm{p}}=5.5 \mathrm{MA}$, which confines alphas very well, and the coils would allow burn times up to $35 \mathrm{~s}$. LHCD is calculated self-consistently using LSC for density profiles with $\mathrm{n}(0) /<\mathrm{n}>\approx 1.5$. This quasi-steady reversed shear discharge with $\mathrm{q}(0)=$ $2.9, \mathrm{q}_{\min }=2.2($ at $\mathrm{r} / \mathrm{a}=0.8)$ and $\mathrm{q}_{95}=3.5$ attained $\beta_{\mathrm{N}}=3.5, \mathrm{f}_{\mathrm{bs}}=64 \%$ and $\mathrm{Q}=7.5$ for moderately enhanced confinement of $\mathrm{H}(\mathrm{y}, 2)=1.6$, and was $100 \%$ non-inductively driven after 11s. The physics based transport code GLF23 predicts the formation of an internal transport barrier (ITB) in FIRE for plasmas with reversed shear and modestly peaked density profiles, $n(0) /\langle n\rangle_{V}=1.5$. Exploitation of these regimes will require stabilization of the $n=1$, either by feedback from coils located in the first wall of the FIRE vacuum vessel or by a method to rotate the FIRE plasma, and improved long pulse capability for the FIRE internal components. An important feature of the FIRE cryogenic copper alloy magnets is that the pulse length increases rapidly as the field is reduced with flattops of $\sim 40 \mathrm{~s}$ at $8 \mathrm{~T}, \sim 90 \mathrm{~s}$ at $6 \mathrm{~T}$ and $240 \mathrm{~s}$ at $4 \mathrm{~T}$. The primary limitation to exploiting this long pulse magnet capability is the generic magnetic fusion problem of handling the plasma exhaust power and nuclear heating of the vacuum vessel under reactor relevant conditions.

\section{Diagnostics}


FIRE will need a comprehensive set of plasma and machine control diagnostics to carry out the mission. A preliminary set of diagnostics has been identified and a tentative assignment of ports has been made [15]. At this point in the design process, it has only been possible to consider conceptual aspects of the integration of diagnostics with the tokamak, its internal hardware and the necessary radiation shielding. There are many diagnostics requiring optical sight-lines to the core plasma and to the divertor, which will require labyrinthine paths through thick shielding "plugs" in the access ducts. Magnetic diagnostics, for measuring parameters such as the plasma current and position and high-frequency instabilities, will necessarily be mounted immediately behind first-wall tiles and must be integrated with the structures planned for these areas. For access to the divertor, and to gain sight-lines for the $\mathrm{x}-$ points and separatrix legs into the divertor and their contact points, significant design integration with the divertor and first wall components is required. A draft R\&D plan for diagnostics has been prepared and will be used to guide requests for diagnostic development.

\section{Engineering Description of FIRE}

The baseline magnetic fields and pulse lengths can be provided by wedged $\mathrm{BeCu} / \mathrm{OFHC}$ toroidal field (TF) coils and OFHC poloidal field (PF) coils that are pre-cooled to $77 \mathrm{~K}$ prior to the pulse and allowed to warm up to $373 \mathrm{~K}$ at the end of the pulse. 3-D finite-element stress analyses of the TF coils which includes electromagnetic, and thermal stress due to ohmic and nuclear heating have shown that this design has a margin of $30 \%$ beyond the usual allowable engineering stress requirements. The present innermost central solenoid coils are near the design stress allowable but have considerable thermal margin. Consequently, consideration is being given to changing the design from OFHC to $\mathrm{Cu}-\mathrm{Cr}-\mathrm{Zr}$ copper alloy. This alloy has an electrical conductivity of $\sim 85 \%$ IACS, but has a yield strength approximately 50\% higher than OFHC copper. FIRE has very little neutron shielding between the plasma and the toroidal field coil, therefore the nuclear heating must be taken into account in the calculation of pulse duration. At 10T, the TF flat top is reduced from $27 \mathrm{~s}$ to $20 \mathrm{~s}$ due to nuclear heating. FIRE, like the previous BPX design, is being designed mechanically to accommodate 3,000 full field, full power pulses and 30,000 pulses at 2/3 field. Neutron damage to the TF insulator limits the total fusion energy production of $5.5 \mathrm{TJ}$ the same as BPX. An insulator R\&D program is proposed that would allow the fusaion enegy to be increased by a factor of $\sim 10$. The repetition time at full field and full pulse length will be $<3 \mathrm{hr}$, with much shorter times at reduced field or pulse length. Large $(1.3 \mathrm{~m}$ by $0.7 \mathrm{~m})$ midplane ports provide access for heating, diagnostics and remote manipulators, while 32 angled ports provide access to the divertor regions for utilities and diagnostics. Remote maintenance inside the vacuum vessel would be accomplished using a cantilevered articulated boom inserted through the large midplane port. The engineering systems are described in more detail by Thome et al $[16,17]$.

FIRE would provide reactor relevant experience for divertor and first wall power handling since the anticipated thermal power densities on the divertor plates of $\sim 6 \mathrm{MWm}^{-2}$ for detached operation, $\sim 12 \mathrm{MW} / \mathrm{m}^{2}$ with modest amounts (few \%) of neon added to the divertor, and a maximum of $\sim 25 \mathrm{MWm}^{-2}$ for attached operation, approach those anticipated for ARIES-RS $[18,19]$. These estimates, based on modeling using the UEDGE Code with edge transport parameters expected for FIRE conditions, result in a power e-folding width of $2.3 \mathrm{~cm} \mathrm{[19].}$ 
The assumptions within UEDGE are tested by comparisons with results on DIII-D and CMod tokamak experiments. The D-T experiments on TFTR and JET observed tritium retention fractions of $\approx 15$ to $30 \%$ with carbon limiters and divertor plates [20]. This large retention fraction would have a significant impact on the operational schedule of a burning plasma experiment and reactor, and would require periodic shutdowns to remove the excess tritium inventory. FIRE would use only reactor relevant metallic materials for plasma facing components, and carbon could not allowed in the vessel due to tritium inventory build-up by co-deposition. The divertor plasma-facing components are tungsten "brush" targets mounted on copper backing plates, similar to a concept developed by the ITER R\&D activity. The tungsten concentration in the plasma core must be kept below $\sim 10^{-5}$ to avoid significant radiation loss. UEDGE modeling shows that the plasma temperature at the divertor target is sufficiently low as to prevent significant sputtering of the tungsten targets [19]. Type I ELMs are also a major concern for a next step burning plasma experiment and fusion reactor operating in the H-mode. ELMS that deposit more than about $1 \mathrm{MJ} / \mathrm{m} 2$ would cause melting of the tungsten, and will limit the lifetime of the divertor so severely that techniques must be found to mitigate the energy deposited by ELMs. There is some evidence that double null operation, high triangularity, and high edge density will reduce the ELM energy deposition [21]. The outer divertor plates and baffle are water-cooled and come into steady-state equilibrium during the pulse [22]. The experimental plan and remote handling system anticipate changing the divertor targets twice during the 15 year operation life of FIRE so accommodate erosion due to ELMs and disruptions.

The first wall is comprised of Be $(5 \mathrm{~mm})$ plasma-sprayed onto copper tiles $[22,23]$. The neutron wall loading at the outboard wall in FIRE is $\sim 2 \mathrm{MWm}^{-2}$ and produces significant nuclear heating of the first wall and vacuum vessel during the $20 \mathrm{~s}$ pulse. The inner divertor targets and first wall are cooled by mechanical attachment to water-cooled copper plates inside the vacuum vessel. Sixteen cryo-pumps - closely coupled to the divertor chambers, but behind sufficient neutron shielding - provide pumping ( $\left.\geq 100 \mathrm{~Pa} \mathrm{~m}^{3} / \mathrm{s}\right)$ for D-T and He ash during the pulse. Pellet injection scenarios with high-field-side launch capability will reduce tritium throughput, and enhance fusion performance. The in-device tritium inventory will be determined primarily by the cycle time of the divertor cryo-pumps, and can range from $<2 \mathrm{~g}$ for regeneration overnight to $\sim 10 \mathrm{~g}$ for weekly regeneration. The tritium usage per shot and inventory is comparable to that of TFTR and therefore will not require a large step beyond previous US fusion program experience in tritium shipping and handling.

The construction cost of the tokamak subsystem (magnets, divertor, plasma facing components and mechanical structure) has been estimated by the FIRE design team and U. S. industry to be $\approx \$ 350 \mathrm{M}$ (FY02US) including $\$ 75 \mathrm{M}$ of contingency. Another $\approx \$ 850 \mathrm{M}$ would be required for auxiliary heating, startup diagnostics, power supplies and buildings to put the project at a new site. If an existing site is used, the site credits would be expected to yield an overall project cost of $\$ 1 \mathrm{~B}(\mathrm{FY} 02 \mathrm{US})$.

\section{Concluding Comments}

FIRE is a natural extension of the existing state of the art tokamaks, and is based on the extensive international $\mathrm{H}$-mode data base for projecting performance to the burning plasma 
regime. Due to the high magnetic field, the extrapolation required to attain $Q \approx 10$ is a modest factor of 3 in terms of the normalized confinement time $\left(B \tau_{\mathrm{E}}\right)$. While this reduces the uncertainty in attaining a burning plasma, it does not extend some plasma parameters (e.g., $\left.\rho^{*}\right)$ to full reactor values. The MHD stability characteristics of FIRE, with $\mathrm{q}_{95} \approx 3.1$ and $\beta_{\mathrm{N}} \approx$ 1.8 for initial burning plasma experiments, are similar to the standard MHD regimes in existing tokamaks and will explore the synergistic effects of energetic alphas and MHD modes such as sawteeth and TAE modes. Operation at $\beta_{\mathrm{N}} \approx 3$ or higher in later phases would begin to explore the important areas of neoclassical tearing modes (NTM) and resistive wall modes (RWM). Lower hybrid current drive and feedback stabilization being evaluated as an experimental tools to investigate the control of NTMs and RWMs. Divertor pumping and pellet fueling will allow FIRE to vary the density, hence the TAE driving terms $R \nabla \beta_{\alpha}$, by a factor of three providing a good test bed for exploring the instability boundary for TAE modes and determining the transport of energetic alpha particles due to multiple overlapping TAE modes.

The double null divertor configuration produces the strongest plasma shaping which is critical for resolving and exploiting a number of beneficial physics effects related to confinement and MHD stability. The double null divertor may also significantly reduce the frequency and intensity of vertical displacement disruptions which is a critical issue for the feasibility of a tokamak based reactor. The high power density in FIRE poses a significant challenge for the divertor and first wall designs, but this is a generic issue that must be solved for magnetic fusion. The success of FIRE in this area would provide important technology development for future fusion devices.

A critical issue for all next step experiments is to supply auxiliary heating power and current drive at high power densities to fusion plasmas. FIRE proposes to use ICRF heating which has been demonstrated on existing experiments, but the high power densities and neutron wall loading present in FIRE will require significant plasma technology R\&D. Similarly, the proposed use of LHCD will require an expansion of the physics and technology R\&D activities in this area. This R\&D will be needed if ICRF and LHCD are to be used in fusion applications.

The FIRE design study is a U. S. national activity managed through the Virtual Laboratory for Technology. The FIRE activities are carried by participants at Advanced Energy Systems, Argonne National Laboratory, Boeing Company, General Atomics, Georgia Instititute of Technology, Idaho National Environmental and Engineering Laboratory, Lawrence Livermore National Laboratory, Los Alamos National Laboratory, Massachusetts Institute for Technology, Oak Ridge National Laboratory, Princeton Plasma Physics Laboratory, Sandia National Laboratory, University of Illinois, and University of Wisconsin. The PPPL work was supported by DOE Contract \# DE-AC02-76CHO3073.

\section{References}

[1] D. M. Meade, et al, Physics Regimes in the Fusion Ignition Research Experiment (FIRE), 
$18^{\text {th }}$ IAEA Fusion Energy Conference, Sorrento, 2000

[2] D. M. Meade, et al, Fusion Ignition Research Experiment (FIRE), Fusion Technology, Vol 39, No 2, part 2, p336 March 2001

[3] D. J. Campbell, The Physics of the International Thermonuclear Experimental Reactor FEAT, Phys. Plasmas, 8 (2001) 2041.

[4] S. C. Jardin, C. E. Kessel, C. G. Bathke, D. A. Ehst, T. K. Mau, F. Najmabadi, and T. W. Petrie, and the ARIES Team, Physics Basis for a Reversed Shear Tokamak Power Plant, Fusion Engineering and Design, Vol. 38, (1997) 27.

[5] M. Kikuchi, Y. Seki and K. Nakagawa, The Advanced SSTR, Fusion Engineering Design 1998

[6] B. Coppi et al, The IGNITOR Experiment and its Perspectives, $16^{\text {th }}$ IAEA Fusion Energy Conference Vol 3, p 579, 1996

[7] D. W. Swain and M.D. Carter, Plasma Heating and Current Drive Systems for the Fusion Ignition Research Experiment (FIRE), this conference.

[8] J. G. Cordey, et al., Energy Confinement in Steady State Elmy H-modes in JET, Proc. $28^{\text {th }}$ EPS Conference on Controlled Fusion and Plasma Physics, 2001, paper 3.11.

[9] J. Kinsey et al, Burning Plasma Confinement Projections Using the GLF23 Transport Model, to be submitted to Fusion Science and Technology.

[10] S. C. Jardin, N. Pomphrey, and J. Delucia, Dynamic modeling of transport and position control of tokamaks, J. Comput. Phys., 66 (1986) 481.

[11] C. D. Warrick, R. J. Buttery, G. Cunningham, et al, Complete stabilization of Neoclassical Tearing Modes with Lower Hybrid Current Drive on COMPASS-D, Phys. Rev. Lett. 85, 574, (2000)

[12] L. C. Johnson, et al, paper P4.008, $28^{\text {th }}$ EPS Conf. on Cont. Fusion and Plasma Physics

[13] J. Bialek, A. H. Boozer, M. E. Mauel, and G. A. Navratil, Modeling of active control of external magnetohydrodynamic instabilities, Phys. Plasmas, 8 (2001) 2170.

[14] C. Kessel et al, Advanced Tokamak Scenarios for the FIRE Burning Plasma Experiment, $19^{\text {th }}$ Symp. Fus. Engineering, January 22-25, 2002, and Physics Basis, and Simulation of Burning Plasma Physics for the Fusion Ignition Research Experiment (FIRE) this conference.

[15] K. Young, et al., Challenges for plasma diagnostics in a next step device (FIRE), $19^{\text {th }}$ Symp. Fus. Engineering, January 22-25, 2002.

[16] R. J. Thome, et al, $21^{\text {st }}$ Symp. on Fusion Technology, Madrid, 2000

[17] R. J. Thome, this conference

[18] M. A. Ulrickson, et al, $21^{\text {st }}$ Symp. on Fusion Technology, Madrid, 2000

[19] D. Driemeyer et al, Design of the Fusion Ignition Research Experiment (Fire) Plasma Facing Components, this conference.

[20] G. Federici, C. H. Skinner, et al, Plasma Material Interactions in Current Tokamaks and their Implications for Next Step Fusion Reactors, Nuclear Fusion, vol. 41, p.1967, Dec 2001

[21] G. Saibene, A. Loarte, R. Sartori, P.J. Thomas, A. Korotkov, R. Koslowski, M. Becoulet, G. Huysmans, G. Sips, G. Conway, Y. Kamada, D. Mossessian, A. Hubbard, T. Leonard, R. Groebner, "High confinement, high density ELMy H-Modes with small ELMs, Presented at EFPW 2001.

[22] M. Ulrickson, et al., Design of Fusion Ignition Research Experiment (FIRE) Plasma Facing Components, 19 ${ }^{\text {th }}$ Symp. Fus. Engineering, January 22-25, 2002. 
[23] R.G. Castro, A.H. Bartlett, K.J. Hollis and R.D. Field, "The effect of substrate temperature on the thermal diffusivity and bonding characteristics of plasma sprayed beryllium," Fus. Eng. \& Design 37 (1996) 243-252. 
Fig. 1. The minimum major radius required to achieve $\mathrm{Q}=10$ in the ELMy $\mathrm{H}$-mode for an inductively driven copper coil tokamak. The coils are precooled to liquid nitrogen temperature and are heated adiabatically to $370 \mathrm{~K}$ by the end of the pulse. The duration of the pulse is specified in plasma current redistribution times, $\tau_{\mathrm{J}}=\tau_{\mathrm{burn}} / \tau_{\mathrm{CR}}$.

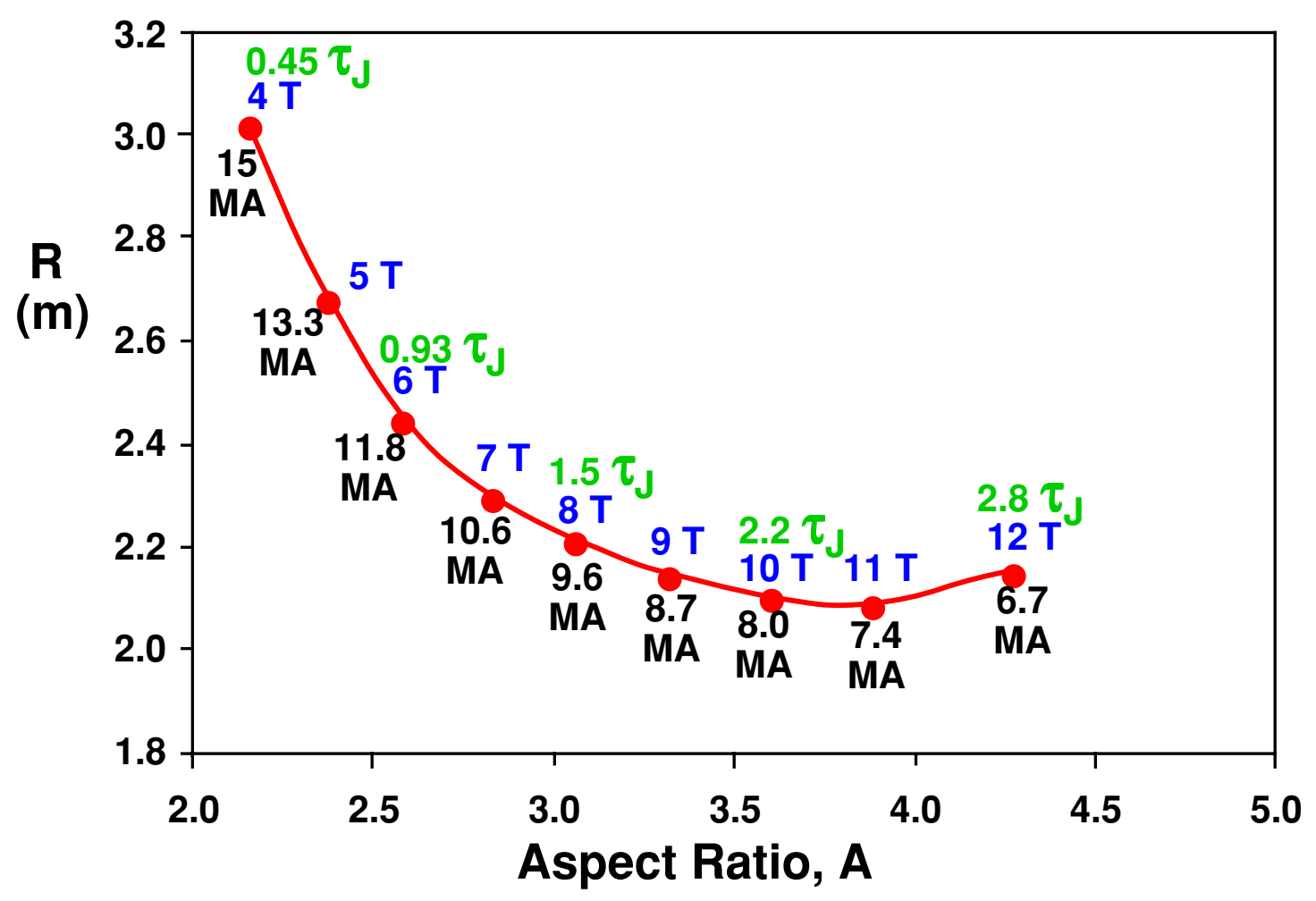


Fig. 2. The cross-section of the FIRE configuration illustrating the major components of the tokamak. The major radius is $2.14 \mathrm{~m}$.

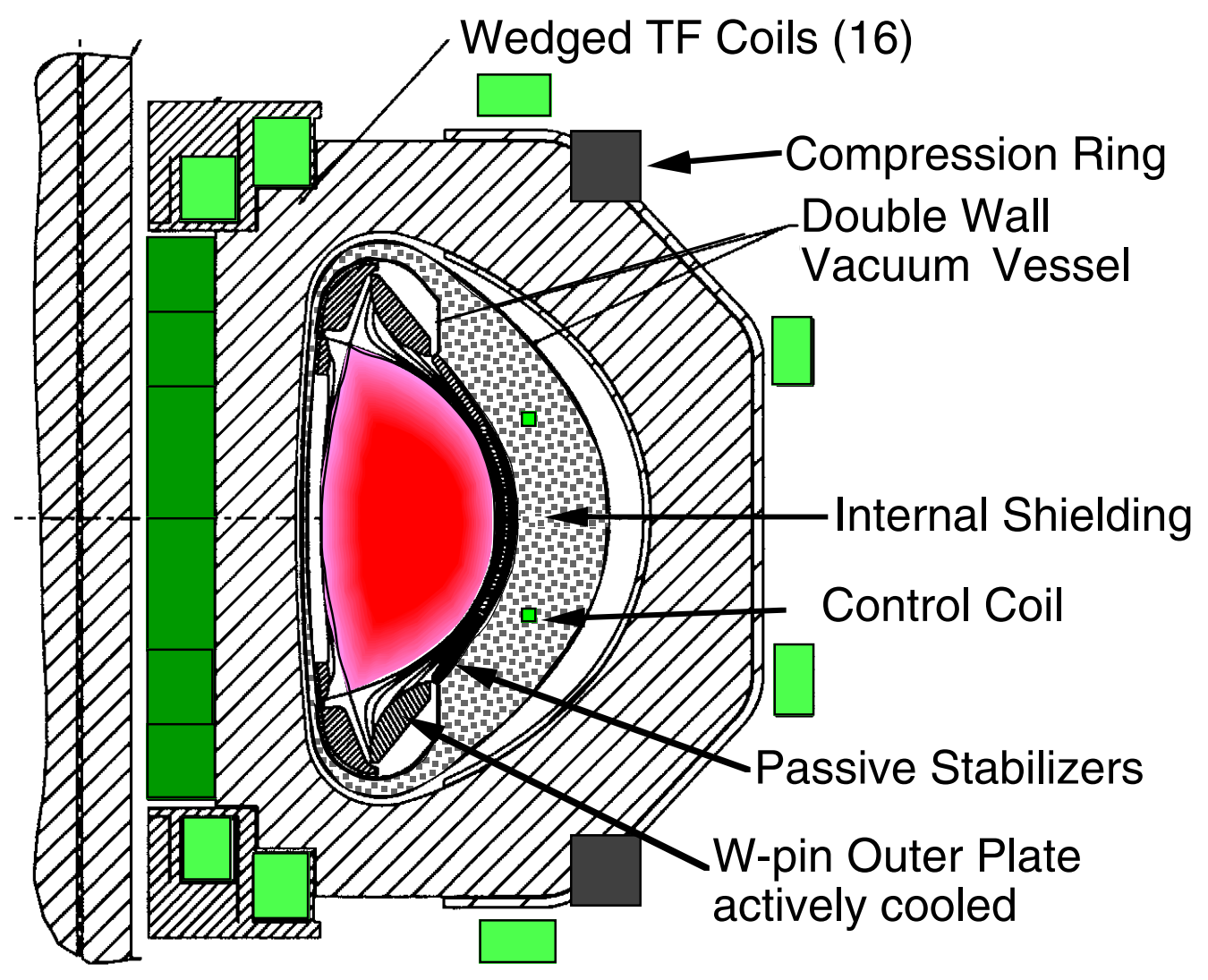


Fig. 3. $\mathrm{H}$ factor for JET H-mode data in the International Data Base DB03v5 selected for FIRE-like conditions of $\beta_{\mathrm{N}}>1.7, \kappa>1.6, Z_{\text {eff }}<2$ and $2.75<\mathrm{q}_{95}<3.5$. The curve was derived by Cordey to fit all data in JET H-mode data base [8]. The standard FIRE case has $\mathrm{H}(\mathrm{y}, 2)=1.1$ which gives $\mathrm{Q}=10$.

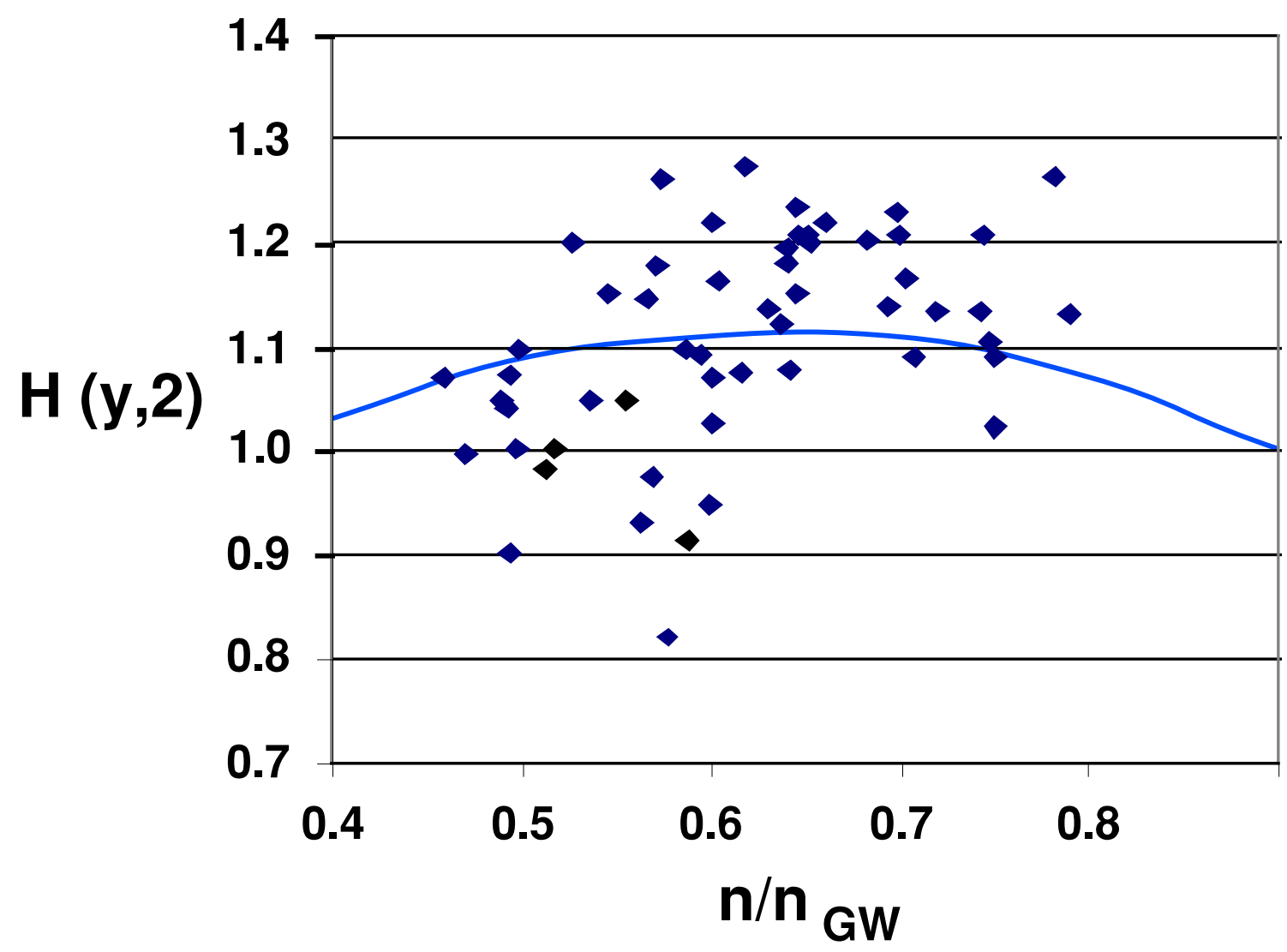


Fig. 4. Sensitivity of fusion gain to small changes in confinement and to density profile peaking. The FIRE base case with $\mathrm{H} 98(\mathrm{y}, 2)=1.1$, and $\mathrm{n}(0) /\langle\mathrm{n}\rangle=1.2$ achieves $\mathrm{Q}=10$. Note, that designs for future tokamak reactors like ARIES-RS or a first reactor based on ITER would require confinement toward the top of the JET H-Mode data base.

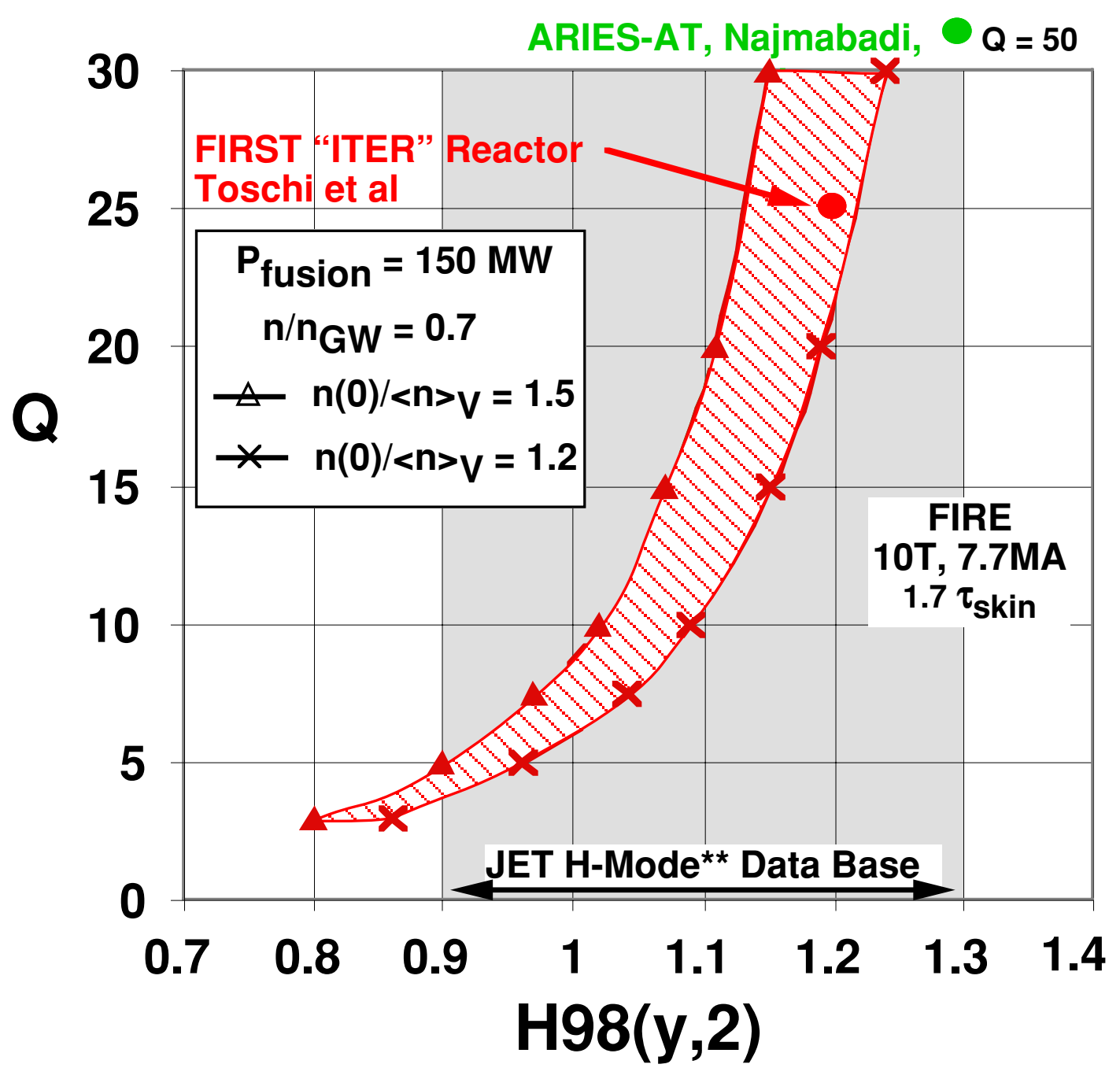


Fig. 5. Simulation of the standard H-mode plasma in FIRE using the Tokamak Simulation Code (TSC) [10]. The plasma density and heating power are programmed to enter the $\mathrm{H}-$ mode during the initiation of plasma burn. The auxiliary power is reduced after the initial thermonuclear burn transient, and the output fusion power is controlled for $\sim 2$ plasma current redistribution times. This simulation has helium ash pumping with $\tau_{\mathrm{He}}=5 \tau_{\mathrm{E}}$. If helium ash pumping is not present, the alpha power decays to $\approx 1 / 3$ by the end of the 20 s pulse.

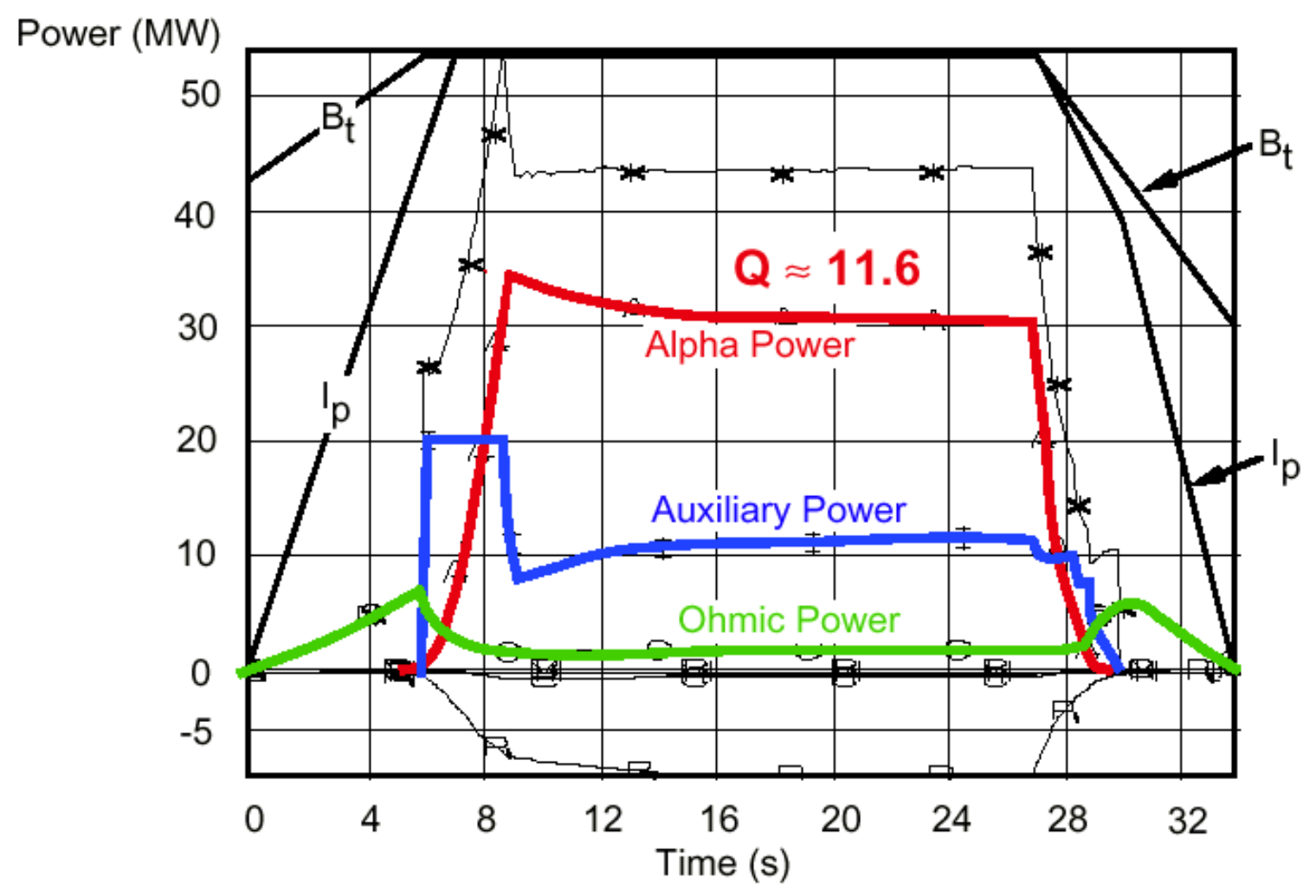


Fig. 6. Simulation of an advanced tokamak (reversed shear) mode in FIRE using TSC. The plasma current rise and minor radius are programmed so that the plasma current profile at the start of flat top $(\sim 10 \mathrm{~s})$ is the same as the steady-state non-inductively driven current profile. This reversed shear discharge with $\mathrm{q}(0)=2.9, \mathrm{q}_{\min }=2.2($ at $\mathrm{r} / \mathrm{a}=0.8)$ and $\mathrm{q}_{95}=3.5$ attained $\beta_{\mathrm{N}}=3.5$ and $\mathrm{f}_{\mathrm{bs}}=64 \%$. The proposed feedback stabilization system would stabilize the resistive wall mode up to $\beta_{\mathrm{N}}=3.8$.

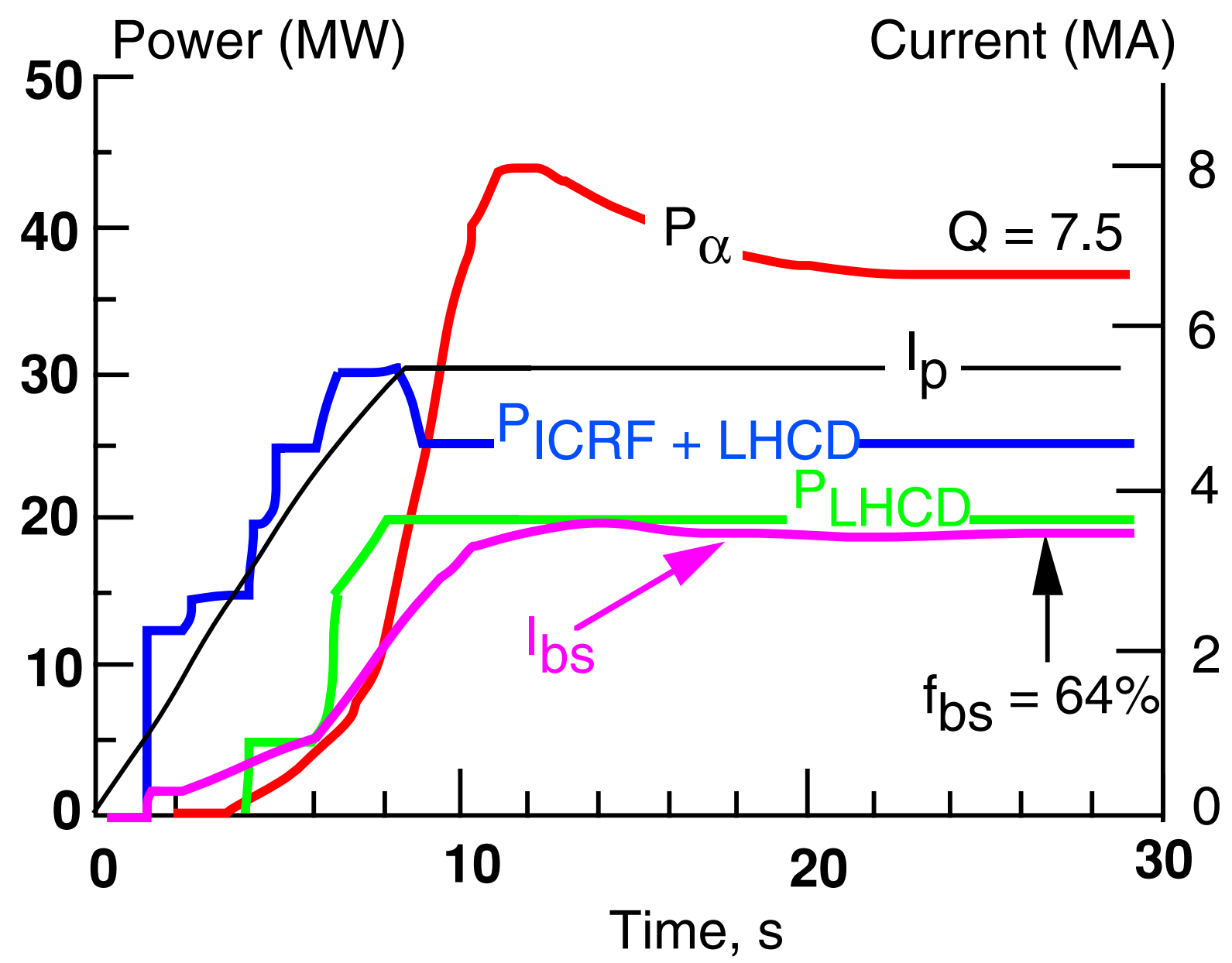


Table I. Typical Parameters for $\mathrm{Q}=10$ in FIRE

\begin{tabular}{|l|c|}
\hline $\mathrm{R}(\mathrm{m}), \mathrm{a}(\mathrm{m})$ & $2.14,0.595$ \\
\hline$\kappa_{\mathrm{x}}, \kappa_{95}$ & $2.0,1.77$ \\
\hline$\delta_{\mathrm{x}}, \delta_{95}$ & $0.7, \approx 0.4-0.55$ \\
\hline $\mathrm{q}_{95}$ & $>3$ \\
\hline $\mathrm{B}_{\mathrm{t}}\left(\mathrm{R}_{\mathrm{o}}\right)(\mathrm{T}), \mathrm{I}_{\mathrm{p}}(\mathrm{MA})$ & $10,7.7$ \\
\hline $\mathrm{n}(0) / / \mathrm{n}\rangle, \mathrm{T}(0) / / \mathrm{T}\rangle$ & $1.2,2.0$ \\
\hline $\mathrm{n} / \mathrm{n}_{\mathrm{GW}}$ & 0.7 \\
\hline $\mathrm{H} 98(\mathrm{y}, 2)$ & 1.1 \\
\hline$\tau_{\mathrm{E}},(\mathrm{s})$ & 1.04 \\
\hline $\mathrm{Z}_{\mathrm{eff}}\left(3 \% \mathrm{Be}+\mathrm{He}\left(5 \tau_{\mathrm{E}}\right)\right)$ & 1.4 \\
\hline $\mathrm{Q}=\mathrm{P}_{\text {fusion }} /\left(\mathrm{P}_{\text {aux }}+\mathrm{P}_{\mathrm{OH}}\right)$ & 10 \\
\hline Fusion Power $(\mathrm{MW})$ & 150 \\
\hline$\beta_{\mathrm{N}}$ & 1.81 \\
\hline $\mathrm{P}_{\text {loss }} / \mathrm{P}_{\mathrm{LH}}$ & 1.3 \\
\hline $\mathrm{R} \nabla \beta_{\alpha}(\%)$ & 3.8 \\
\hline Fusion Power Density $\left(\mathrm{MWm}^{-3}\right)$ & 5.8 \\
\hline Neutron Wall Loading $\left(\mathrm{MWm}^{-2}\right)$ & 2 \\
\hline
\end{tabular}




\section{External Distribution}

Plasma Research Laboratory, Australian National University, Australia

Professor I.R. J ones, Flinders University, Australia

Professor J oão Canalle, Instituto de Fisica DEQ/IF - UERJ , Brazil

Mr. Gerson O. Ludwig, Instituto Nacional de Pesquisas, Brazil

Dr. P.H. Sakanaka, Instituto Fisica, Brazil

The Librarian, Culham Laboratory, England

Library, R61, Rutherford Appleton Laboratory, England

Mrs. S.A. Hutchinson, JET Library, England

Professor M.N. Bussac, Ecole Polytechnique, France

Librarian, Max-Planck-Institut für Plasmaphysik, Germany

J olan Moldvai, Reports Library, MTA KFKI-ATKI, Hungary

Dr. P. Kaw, Institute for Plasma Research, India

Ms. P.J . Pathak, Librarian, Insitute for Plasma Research, India

Ms. Clelia De Palo, Associazione EURATOM-ENEA, I taly

Dr. G. Grosso, Instituto di Fisica del Plasma, Italy

Librarian, Naka Fusion Research Establishment, J AERI, J apan

Library, Plasma Physics Laboratory, Kyoto University, J apan

Research Information Center, National Institute for Fusion Science, J apan

Dr. O. Mitarai, Kyushu Tokai University, J apan

Library, Academia Sinica, Institute of Plasma Physics, People's Republic of China

Shih-Tung Tsai, Institute of Physics, Chinese Academy of Sciences, People's Republic of China

Dr. S. Mirnov, TRINITI, Troitsk, Russian Federation, Russia

Dr. V.S. Strelkov, Kurchatov Institute, Russian Federation, Russia

Professor Peter Lukac, Katedra Fyziky Plazmy MFF UK, Mlynska dolina F-2, Komenskeho Univerzita, SK-842 15 Bratislava, Slovakia

Dr. G.S. Lee, Korea Basic Science Institute, South Korea

Mr. Dennis Bruggink, Fusion Library, University of Wisconsin, USA

Institute for Plasma Research, University of Maryland, USA

Librarian, Fusion Energy Division, Oak Ridge National Laboratory, USA

Librarian, Institute of Fusion Studies, University of Texas, USA

Librarian, Magnetic Fusion Program, Lawrence Livermore National Laboratory, USA

Library, General Atomics, USA

Plasma Physics Group, Fusion Energy Research Program, University of California at San Diego, USA

Plasma Physics Library, Columbia University, USA

Alkesh Punjabi, Center for Fusion Research and Training, Hampton University, USA

Dr. W.M. Stacey, Fusion Research Center, Georgia Institute of Technology, USA

Dr. J ohn Willis, U.S. Department of Energy, Office of Fusion Energy Sciences, USA

Mr. Paul H. Wright, Indianapolis, Indiana, USA 
The Princeton Plasma Physics Laboratory is operated by Princeton University under contract with the U.S. Department of Energy.

\author{
Information Services \\ Princeton Plasma Physics Laboratory \\ P.O. Box 451 \\ Princeton, NJ 08543
}

Phone: 609-243-2750

Fax: 609-243-2751

e-mail: pppl_info@pppl.gov

Internet Address: http://www.pppl.gov 